\title{
Editorial
}

\section{The laryngeal mask airway in anaesthesia}

The most frequent serious adverse respiratory events associated with anaesthesia are inadequate ventilation, oesophageal intubation and difficult intubation. ${ }^{1} \mathrm{~A}$ clear upper airway is essential for gas exchange - oxygenation, removal of carbon dioxide, and uptake and elimination of inhaled anaesthetics. This is often achieved for short operative procedures with a face mask and oropharyngeal airway, pulling up on the chin and, if necessary, thrusting the jaw forwards. A clear airway may be difficult to achieve or maintain in some patients because, although the Guedel airway prevents the tongue from falling back against the posterior pharyngeal wall, recent research suggests that this is not the only problem. A reduction in tone occurs in all pharyngeal structures during general anaesthesia and obstruction may occur at several sites. ${ }^{2,3}$ Furthermore, face masks are of standard shape and an airtight seal may be impossible to achieve in edentulous or bearded patients and in those with abnormal facial contours. Face mask anaesthesia in these patients is unsatisfactory because it is difficult and tiring, it occupies both hands of the anaesthetist, and it cannot be depended upon to provide an airtight circuit for positive-pressure ventilation.

Passage of a cuffed tracheal tube ensures an airtight circuit so that the patient's inspired gases are not diluted by room air during spontaneous respiration, contamination of the operating room air is minimized and positivepressure ventilation can be used when necessary. Although protection of the lungs from gastric contents is important, in some cases over-riding importance, the commonest reason for passage of a tracheal tube in healthy, elective patients is the effortless maintenance of a clear airway. The technique was pioneered before the First World War although, until the introduction of muscle relaxants, it was often time-consuming and difficult and was only practised by a few specialist anaesthetists. ${ }^{4}$ Because of this, Leech designed the pharyngeal bulb gasway $^{5}$ in Regina in 1935 to provide a clear airway with an airtight seal for closed circuit cyclopropane anaesthesia

From the Department of Anaesthesia, Foothills Hospital and the University of Calgary, 1403 - 29 Street NW, Calgary, Alberta T2N 2 T9.
J. Roger Maltby MB FRCA FRCPC

without intubation. He made wax casts of cadaver throats of men and women and, from these, made a resilient soft rubber bulb that fitted over an oropharyngeal airway and conformed to the contours of the pharynx. Under surgical anaesthesia, the lubricated device was passed over the tongue, following the roof of the mouth, into the pharynx. He attributed any initial leakage of air around the bulb to inadequate relaxation of the pharyngeal and tongue muscles that was corrected by deepening the anaesthesia. The device was used for operations on the face, nose, ears, head and neck; dental procedures but not tonsillectomy; and in patients in the prone position.

The introduction of succinylcholine in the $1950 \mathrm{~s}^{8}$ made tracheal intubation so quick and easy that the technique eventually came to be used for all but the shortest anaesthetics. Major complications are now rare in the hands of skilled and experienced anaesthetists, and its advantages far outweigh its dangers in most patients. Nevertheless, postoperative sore throat and succinylcholineinduced myalgia are common, teeth may be damaged, broken or inhaled; ${ }^{7}$ and unexpectedly difficult intubations still occur despite scoring systems designed to predict such problems. ${ }^{8}$ Pulmonary aspiration of gastric contents may occur if gagging and regurgitation are provoked, ${ }^{9}$ either when the effect of succinylcholine wears off before the trachea is intubated, or at extubation. If tracheal intubation is difficult, the oesophagus may be intubated by mistake, or the anaesthetist may be unable to restore a clear airway to ventilate the patient's lungs.

The cardiovascular stress response to laryngoscopy in adults includes hypertension, tachycardia and arrhythmias which may be prevented or treated with intravenous lidocaine, sodium nitroprusside, nitroglycerin, hydralazine, esmolol or opioids. ${ }^{7}$ Furthermore, the response of the highly sensitive trachea to the presence of a foreign body requires the use of muscle relaxants, or a greater depth of anaesthesia or higher doses of opioids, than might be required for the surgical procedure. Pharmacological suppression of these responses means that drugs are required, or their dosage increased, to allow the patient to tolerate the tracheal tube rather than to provide better operating conditions for the surgeon. 
The laryngeal mask airway ${ }^{10}$ represents a new concept in airway management. Brain has documented its evolution and development from rubber and latex prototypes to the silicone versions " that became commercially available in 1988. The inflatable boat-shaped cuff at the distal end of the tubular portion forms an end-to-end anastomosis around the larynx. The device can be placed blindly without the aid of muscle relaxants and provokes less reflex response than does tracheal intubation. It may also provide a clear airway in case of failed or predictably difficult intubation. Correct placement of the laryngeal mask airway provides a seal that is more efficient than that of a face mask and equal to that of a tracheal tube with ventilating pressures up to $20 \mathrm{~cm}$ water, with minimal audible leak into the stomach or to the atmosphere, ${ }^{12,13}$ and can be used with low-flow or closed circuit anaesthesia.

In this issue of the Journal, Asai and Morris ${ }^{14}$ provide a comprehensive review of this revolutionary airway device. Opinions on the range of surgical procedures for which the laryngeal mask airway is suitable vary among anaesthetists. Before the laryngeal mask airway became available, indications for tracheal intubation were broadly classified as mandatory (intracranial, intrathoracic, major head and neck, major surgery in prone position, intraoral surgery including tonsillectomy, intestinal distension and any patient with a full stomach); preferable (upper abdominal, positions that compromise respiratory physiology); optional (lower abdominal, minor head and neck); not justified (minor gynaecological and urological, limbs, superficial trunk). ${ }^{15}$ Some anaesthetists believe the laryngeal mask airway should only be used in the last category. Asai and Morris document its use as an alternative to tracheal intubation, not only for surgery on the limbs and trunk but also, surprisingly, for laparoscopy, dental surgery, tonsillectomy, and for patients in the lateral or prone position.

Safe use of the laryngeal mask airway for these latter procedures should cause us to reconsider indications for tracheal intubation in terms of purpose 7,16 rather than by lists of surgical procedures. Indications in order of frequency in elective patients would be effortless maintenance of a clear airway, use of positive-pressure ventilation, non-interference with the surgical field, and protection of the airway from gastric contents. When the laryngeal mask airway is correctly placed the first three requirements are fulfilled but not the fourth. Its use is therefore contraindicated in the presence of aspiration risk factors, especially a full stomach or gastro-oesophageal reflux, except as a temporizing measure in failed intubation. Several cases of pulmonary aspiration, some of which were severe, ${ }^{18}$ have been reported but none was fatal in an estimated usage of several million. ${ }^{19}$ The in- cidence of clinically important pulmonary aspiration in healthy elective patients before introduction of the laryngeal mask was approximately 1 in $10,000 .{ }^{9}$ There have been no randomized, prospective studies that compare the safety of the laryngeal mask airway with tracheal intubation in similar patient populations.

High pulmonary inflation pressures at present require the presence of a cuffed tracheal tube, although a high pressure seal laryngeal mask airway is in the development stage. Since the laryngeal mask airway lies in the hypopharynx and does not enter the trachea, it should not be used when local pathology is present that could cause respiratory obstruction. Tumour, abscess, haematoma or oedema in the pharynx, larynx or trachea, and compression of the trachea by an enlarged thyroid gland or mediastinal tumour are usually indications for passage of a tracheal tube under local anaesthesia before general anaesthesia is induced.

Numerous case reports testify to the successful use of the laryngeal mask airway in both emergency and elective cases in which intubation, and sometimes ventilation, was impossible. These include Caesarean section, fractured cervical spine, Pierre-Robin syndrome and even one case of open-heart surgery. In all these cases, the presence of a cuffed tracheal tube was desirable, if not "mandatory" to guarantee a clear, protected airway. However, the outcomes suggest that there are no absolute rules in these situations. The laryngeal mask airway does not protect against pulmonary aspiration of gastric contents but, when intubation is impossible, nor does a cuffed tracheal tube that is still in the anaesthetist's hand. Patients do not die from not having a tube in the trachea, they die from hypoxia due to inadequate ventilation. They must receive oxygen by some means. The laryngeal mask airway may establish a clear airway to buy time until the patient awakens, to act as a guide for blind tracheal intubation or to facilitate use of a fibreoptic bronchoscope or tube exchanger. The anaesthetist may also pause to consider whether intubation is really necessary because airway problems may also occur following extubation when rapid reintubation is unlikely to be easy. Therefore, if the laryngeal mask airway establishes a clear airway with no leak, it may sometimes be appropriate to proceed without tracheal intubation and allow the patient to awaken with the laryngeal mask airway in place. If tracheal intubation is performed through the laryngeal mask airway, the latter can be left in place during the procedure to provide a clear airway after extubation, or it can be reinserted at that time. When a difficult airway is anticipated, the laryngeal mask airway can be inserted with topical and its correct position confirmed before general anaesthesia is induced or one of the above alternatives chosen. 
Complications associated with tracheal intubation are now rarely reported; they are so well known that they are regarded as the inevitable accompaniment of a technique essential for the safety of anaesthetized patients. Letters to the editor and case reports continue to document both benefits and complications associated with use of the laryngeal mask airway. There have been few randomized prospective studies ${ }^{20-23}$ that compare the safety, efficacy and morbidity of the laryngeal mask airway with either tracheal intubation or face mask/oropharyngeal airway. None has compared all three techniques for the same surgical procedure such as arthroscopy. Such a study should also include cost analysis to determine whether the high initial cost of the reusable laryngeal mask airway is offset by savings on disposable tracheal tubes, pharmacy costs and recovery room nurses' time devoted to holding patients' chins.

The reinforced laryngeal mask airway is particularly useful in head and neck surgery because the more flexible tubular portion may be turned in any direction close to the face without altering the position of the cuff around the larynx. Other prototypes that are undergoing clinical evaluation include an intubating laryngeal mask airway and one with a high pressure seal with a posterior tube for passage of a gastric tube.

Tracheal intubation is usually easy and atraumatic, but not always. Just because we are able to intubate the trachea does not mean that we should do so indiscriminately. perméabilité des voies aériennes supérieures permet les échanges respiratoires: l'oxygénation, l'élimination du gaz carbonique, la capture et l'élimination des agents anesthésiques inhalatoires. Souvent un masque facial et une canule oropharyngée avec traction du menton vers le haut, et si nécessaire poussée de la mâchoire vers l'avant sont suffisants. Chez certains patients, la liberté des voies aériennes supérieures est difficile à réaliser et à maintenir; bien que la canule de Guedel prévienne la chute vers l'arrière de la langue contre le mur laryngé postérieur, des études récentes suggèrent que ce problème n'est pas le seul. Pendant l'anesthésie générale, le tonus de toutes les structures pharyngées s'affaisse et une obstruction peut apparaître à plusieurs endroits. ${ }^{2,3}$ De plus les masques faciaux ont tous une forme identique et l'étanchéité à l'air peut s'avérer impossible chez l'édenté, le barbu ou chez celui dont le contour facial est anormal. L'anesthésie au masque facial garde occupées les deux mains de l'anesthésiste et ne procure pas les conditions d'étanchéité essentielles à la ventilation en pression positive; dans ces cas, elle devient inefficace et pénible.

La mise en place d'un tube trachéal à manchette assure l'étanchéité du circuit; en ventilation spontanée, les gaz. inspirés par le patient ne sont pas dilués par l'atmosphère, la contamination gazeuse de la salle d'opération est réduite et, au besoin, on peut passer à la ventilation mécanique. Il est important et même, dans certains cas, absolument essentiel de protéger le voumon contre le
En anesthésie, l'incapacité de ventiler, l'intubation oesophagienne et l'intubation difficile représentent les problèmes respiratoires les plus souvent rencontrés. ${ }^{1} \mathrm{La}$ de la face, du nez, des oreilles, de la tête et du cou; les interventions dentaires à l'exception des amygdalectomies; et enfin pour le décubitus ventral. 
En 1950, l'introduction de la succinylcholine rend l'intubation si facile et si rapide qu'on se met même à la pratiquer pour les anesthésies très courtes. Avec des anesthésistes habiles et expérimentés, les complications sont très rares et, pour la plupart du temps, ses avantages l'emportent largement sur ses inconvénients. Néanmoins, les maux de gorge causés par les manipulations et les myalgies induites par la succinylcholine sont fréquents; les dents peuvent être endommagées, brisées et aspirées; ${ }^{7}$ et, hors de toute attente, et malgré des méthodes d'évaluation conçues pour prédire ce genre de problèmes, les intubations difficiles sont toujours à craindre. ${ }^{8}$ Un hautle-coeur ou une régurgitation peuvent provoquer l'aspiration du contenu gastrique, ${ }^{9}$ soit avant l'intubation, lorsque l'activité de la succinylcholine décline, soit à l'extubation. En cas d'intubation trachéale difficile, on peut intuber l'oesophage accidentellement et il peut aussi devenir impossible pour l'anesthésiste rétablir la filière des voies aériennes et de ventiler les poumons.

Chez l'adulte, la laryngoscopie provoque des réactions cardio-vasculaires: il faut prévenir ou traiter l'hypertension, la tachycardie et les arythmies avec de la lidocaïne, du nitroprussiate de soude, de la nitroglycérine, de l'hydralazine, de l'esmolol et des morphiniques. ${ }^{7}$ De plus, l'hypersensibilité trachéale à lá présence d’un corps étranger nécessite l'usage de myorelaxants, une anesthésie plus profonde ou des doses plus importantes de morphiniques ${ }^{7}$ que celles qu'on utiliserait pour l'intervention chirurgicale elle-même. La suppression de ce type de réponse nécessite donc l'utilisation de drogues, ou l'augmentation des posologies afin de mieux tolérer un tube trachéal et non pour procurer de meilleures conditions pour la chirurgie.

Le masque laryngé $e^{10}$ représente un nouveau concept adapté à la prise en charge des voies aériennes. Brain a décrit son développement et son évolution, à partir de prototypes en latex jusqu'aux versions " en silicone disponibles depuis 1988. La manchette gonflable en forme de navire située à la partie distale de la portion tubulaire crée une anastomose termino-terminale avec le larynx. L'appareil peut être inséré à l'aveugle sans recours de myorelaxants et provoque moins de réflexes que l'intubation à l'aveugle. Il peut assurer la perméabilité des voies aériennes en cas d'intubation impossible ou ratée. Son installation correcte procure une étanchéité et une efficacité supérieures à celles du masque facial ordinaire et équivaut au tube trachéal lorsque les pressions ventilatoires sont moindres que $20 \mathrm{~cm} \mathrm{H}_{2} \mathrm{O}$ avec une fuite gazeuse minime dans l'estomac ou l'atmosphère; ${ }^{12,13}$ il peut être utilisé avec des bas débits ou en circuit fermé.

Vous lirez ce mois-ci l'article d'Asai et Morris ${ }^{14}$ qui examine en détail ce type révolutionnaire de canule. Les anesthésistes ne s'entendent pas entre eux sur la gamme d'interventions pour lesquelles l'instrument est utilisable.
Avant l'introduction du masque laryngé, on classifiait les indications de lintubation en obligatoires (interventions intracrâniennes, intrathoraciques, cervico-céphaliques majeures, la position ventrale, la chirurgie intrabuccale dont l'amygdalectomie, la distension abdominale et toutes les interventions avec estomac plein); en préférables (interventions abdominales supérieures, toutes les positions qui mettent en danger la perméabilité des voies aériennes); en optionnelles (interventions abdominales, cervicocéphaliques mineures); injustifiées (chirurgie gynécologique et urologique mineures, périphérique et tronculaire mineure). ${ }^{15}$ Certains anesthésistes croient que le masque laryngé ne devrait pas être utilisé pour les interventions de cette dernière catégorie. Asai et Morris regardent son utilisation comme une alternative à l'intubation trachéale, non seulement pour la chirurgie des membres et du tronc, mais aussi, chose surprenante, pour la laparoscopie, la chirurgie dentaire, l'amygdalectomie et chez des patients en position latérale et ventrale.

L'utilisation du masque laryngé pour ces dernières interventions nous force à reconsidérer les objectifs de l'intubation trachéale ${ }^{7,16}$ plutôt que son attribution à des listes dinterventions. Par ordre de fréquence, les indications de l'intubation en chirurgie réglée devraient inclure la facilité de la prise en charge des voies aériennes, l'utilisation de la ventilation mécanique, l'absence d'intrusion dans le champ opératoire, et la protection des voies respiratoires contre l'aspiration du contenu gastrique. Avec un masque laryngé bien assis les trois premières conditions sont remplies, mais non la quatrième. Son utilisation est donc.contre-indiquée en présence de facteurs de risque d'aspiration, spécialement l'estomac plein ou le reflux gastro-oesophagien, et exceptionnellement, comme palliatif à l'intubation ratée. Plusieurs cas d'aspiration pulmonaire, quelquefois grave ${ }^{16}$ ont été rapportés mais aucune n'a été fatale sur un total de plusieurs millions d'utilisation. ${ }^{19}$ Lincidence d'aspiration clinique importante pour une population bien portante en chirurgie non urgente avant l'apparition du masque laryngé est estimée à 1 sur $10,000 .{ }^{9}$ Nous ne possédons pas à l'heure actuelle d'études randomisées prospectives qui comparent chez des populations identiques la sécurité du masque laryngé avec l'intubation.

Des pressions élevées d'insufflation appliquées au poumon nécessitent la présence d'une canule trachéale avec manchette, bien qu'un masque laryngé étanche à haute pression soit en voie de développement. Comme le masque laryngé repose dans l'hypopharynx et ne pénètre pas dans la trachée, il ne devrait pas être utilisé lorsqu'une pathologie locale est susceptible de causer de l'obstruction. Les tumeurs, abcès, hématomes ou oedèmes pharyngés, laryngés ou trachéaux et les compressions trachéales extrinsèques causées par l'hypertrophie de la thyroïde 
ou une tumeur de médiastin sont ordinairement des indications pour l'introduction d'une canule trachéale sous anesthésie locale avant l'induction de l'anesthésie générale.

On rapporte plusieurs observations qui témoignent de l'usage heureux du masque laryngé dans des situations urgentes et non urgentes où l'intubation et même la ventilation étaient impossibles. Il faut retenir, entre autres, la césarienne, la fracture cervicale, le syndrome de Pierre Robin et même un cas de chirurgie cardiaque à coeur ouvert. Dans tous ces cas, l'intubation trachéale était désirable, sinon obligatoire pour maintenir la perméabilité et assurer la protection des voies aériennes. Toutefois, les résultats suggèrent qu'il n'existe pas, dans ces situations, de règles absolues. Le masque laryngé ne protège pas de l'aspiration du contenu gastrique, mais, lorsque l'intubation est impossible, le tube trachéal qui demeure dans la main de l'anesthésiste, lui non plus. Les malades ne décèdent pas de ne pas avoir un tube dans la trachée, mais bien d'hypoxie par insuffisance ventilatoire. Il doivent recevoir de l'oxygène par un méthode ou une autre. La masque laryngé peut garder les voies aériennes perméables pour sauver du temps jusqu'au réveil du patient, pour servir de guide à lintubation trachéale à l'aveugle, ou favoriser la fibroscopie ou une sonde permettant l'échange de canule. L'anesthésiste peut de plus prendre une pause et sinterroger sur l'absolue nécessité de l'intubation parce des problèmes de voies aériennes peuvent aussi survenir après l'extubation alors que la réintubation rapide risque aussi d'être difficile. En conséquence, si le masque laryngé établit la filière des voies aériennes sans fuite d'air, il sera quelquefois légitime de continuer sans intuber et de permettre au patient de s'éveiller avec la masque laryngé en place. Si l'intubation trachéale est réalisée à travers le masque laryngé, ce dernier peut être laissé en place pendant l'intervention pour assurer la perméabilité des voies respiratoires à l'extubation, ou encore il pourra être réintroduit à ce moment. Quand on anticipe des difficultés d'intubation, le masque laryngé peut être inséré sous anesthésie topique et sa position confirmée avant l'induction de l'anesthésie générale; on pourra aussi choisir une des alternatives déjà proposées.

On rapporte maintenant rarement des complications avec l'intubation trachéale; elles sont si bien connues qu'elles sont considérées comme des séquelles inévitables d'une technique essentielle à la sécurité des patients anesthésiés. Des lettres à la rédaction et des observations continuent de documenter les bénéfices et complications aśsociées au masque laryngé. On trouve toutefois peu d'études prospectives et randomisées ${ }^{20-23}$.comparant la sécurité, l'efficacité et la morbidité du masque laryngé avec l'intubation trachéale ou le masque facial avec canule oropharyngée. Aucune des études n'a comparé ces trois techniques pour une intervention comme l'arthroscopie. Toute étude de ce type devrait aussi inclure l'analyse des coûts pour déterminer si le coût initial du masque laryngé réutilisable est compensé par des économies pour les tubes à usage unique, les coût de la pharmacie et les dépenses occasionnées par le travail des infirmières de la salle de réveil qui doivent tenir le menton des patients.

Le masque laryngé avec tubulure renforcée est surtout utile pour la chirurgie de la tête et du cou, parce que sa partie tubulaire peut être dirigée dans toutes les directions sans qu'on ait à modifier la position de la manchette installée sur le larynx. D'autres prototypes sont soumis à des évaluations cliniques dont un masque laryngé pour intubation trachéale et un autre doté d'une manchette postérieure à haute pression permettant la mise en place d'une canule gastrique.

L'intubation trachéale n'est pas toujours facile et atraumatique mais le seul fait qu'on puisse intuber facilement ne justifie pas son usage à la légère. La même remarque s'applique au masque laryngé. Cet appareil peut présenter des dangers non négligeables et quand son insertion devient difficile, vaut mieux intuber. Par contre, dans une situation où l'intubation et la ventilation sont impossibles, le masque laryngé peut devenir une bouée de sauvetage. Toute indication pour une de ces techniques doit mettre en balance la sécurité et l'efficacité contre la morbidité. Maintenant que nous disposons d'une alternative moins effractive de prise en charge des voies aériennes, l'anesthésiste n'a plus besoin d'avoir recours à l'intubation trachéale pour assurer son confort personnel.

\section{References}

1 Caplan RA, Posner KL, Ward RJ, Cheney FW. Adverse respiratory events in anesthesia: a closed claims analysis. Anesthesiology 1990; 72: 828-33.

2 Drummond GB. "Keep a clear airway." (Editorial). Br J Anaesth 1991; 66: 153-6.

3 Nandi PR, Charlesworth CH, Taylor SJ, Nunn JF, Doré CJ. Effect of general anaesthesia on the pharynx. $\mathrm{Br} \mathrm{J}$ Anaesth 1991; 66: 157-62.

4 Griffith HR. The boundless realm of anaesthesiology. Can Med Assoc J 1960; 82: 859-65.

5 Leech $B C$. The pharyngeal bulb gasway: a new aid in cyclopropane anesthesia. Anesth Analg 1937; 16: 22-5.

6 Von Dardel O, Thesleff $S$. Succinylcholine iodide as a muscular relaxant. A report of 500 surgical cases. Acta Chirurgica Scandinavica 1952; 103: 321-36.

7 Stone DJ, Gal TJ. Airway management. In: Miller RD (Ed.). Anaesthesia, 3rd ed. New York: Churchill Livingstone 1990; 1265-92.

8 Rose DK, Cohen MM. The airway: problems and predictions in 18,500 patients. Can J Anaesth 1994; 41: 372-83.

9 Warner MA, Warner ME, Weber JG. Clinical significance 
of pulmonary aspiration during the perioperative period. Anesthesiology 1993; 78: 56-62.

10 Brain AIJ. The laryngeal mask - a new concept in airway management. Br J Anaesth 1983; 55: 801-5.

11 Brain $A I J$. The development of the laryngeal mask - a brief history of the invention, early clinical studies and experimental work from which the laryngeal mask evolved. Eur J Anaesthesiol 1991; Suppl 4: 5-17.

12 White $D C$. The laryngeal mask - a non-invasive airway. Eur J Anaesthesiol 1991; Suppl 4: 1-4.

13 Devitt $J H$, Wenstone $R$, Noel $A G$, O'Donnell MP. The laryngeal mask airway and positive-pressure ventilation. Anesthesiology 1994; 80: 550-5.

14 Asai T, Morris $S$. The laryngeal mask airway: its features, effects and role. Can J Anaesth 1994; 41: 930-60.

15 Collins VJ. Principles of Anesthesiology, 2nd ed. Philadelphia: Lea \& Febiger: 1976: 378.

16 Stehling LC. Management of the airway. In: Barash PG, Cullen BF, Stoelting RK (Eds.). Clinical Anesthesia, 2nd ed: Philadelphia: J.B. Lippincott Company 1992; 685-708.

17 Young TM. The laryngeal mask in dental anaesthesia. Eur J Anaesthesiol 1991; 4: 53-9.

18 Nanji GM, Maltby JR. Vomiting and aspiration pneumonitis with the laryngeal mask airway. Can J Anaesth 1992; 39: 69-70.

19 Brimacombe J, Berry A. Aspiration and the laryngeal mask airway - a survey of Australian intensive care units (Letter). Anaesth Intensive Care 1992; 20: 534-5.

20 Smith I, White PF. Use of the laryngeal mask airway as an alternative to a face mask during outpatient arthroscopy. Anesthesiology 1992; 77: 850-5.

21 Webster AC, Morley-Forster PK, Dain S, et al. Anaesthesia for adenotonsillectomy: a comparison between tracheal intubation and the armoured laryngeal mask airway. Can J Anaesth 1993; 40: 1171-7.

22 Williams PJ, Bailey PM. Comparison of the reinforced laryngeal mask airway and tracheal intubation for adenotonsillectomy. Br J Anaesth 1993; 70: 30-3.

23 Swann DG, Spens H, Edwards SA, Chestnut RJ. Anaesthesia for gynecological laparoscopy - a comparison between the laryngeal mask airway and tracheal intubation. Anaesthesia 1993; 48: 431-4. 